\title{
Mobile Electronic Memos
}

\author{
Giovanni Bartolomeo $^{1}$, Stefano Salsano ${ }^{1}$ and Antonella Frisiello ${ }^{2}$ \\ ${ }^{1}$ University of Rome Tor Vergata, via del Politecnico, 1 \\ 00133 Rome, Italy \\ \{Giovanni.Bartolomeo, Stefano.Salsano\}@Uniroma2.it \\ ${ }^{2}$ Istituto Superiore Mario Boella, via Boggio, 61 \\ 10138 Turin, Italy \\ Frisiello@ISMB.it
}

\begin{abstract}
This paper explores a novel concept called Mobile Electronic Memo whose purpose is to overcome the limitations in terms of interoperability and accessibility imposed by the current technology. To achieve this goal, Mobile Electronic Memos decouple the semantics of the information from the physical and pragmatic constraints of the medium that transports it. We examine related works and standards that could be good candidates for implementing MEMs and describe a prototype implementation that has been realized as a proof of concept.
\end{abstract}

Keywords: Electronic information; interoperability; accessibility.

\section{Introduction}

Often the information one needs is already available, somewhere, in an electronic format. In most cases, the information is downloadable from the Internet; or it could be gathered from nearby devices, from the environment (e.g., through sensors) or even from real world objects (e.g., using RF-IDs and QR codes). Once obtained, this information could be stored on consumer electronics (computing devices, mass storages, memory cards), or be shared on the Web. It could be also reused and transmitted asynchronously over a long distance through emails or multimedia messages; or it might be sent as an input to other devices or applications (navigators, ATMs and vending machines, home or car appliances). Zillions of information are daily exchanged this way; but, in many cases, interoperability between the device providing an information and the one consuming it is not automatic, but it is mediated by human intervention, often involving manual inputs.

Unfortunately, this well known limitation might cut off several categories of people from the benefits of the ICT, resulting in a possible reduction of the inclusion opportunities (one aspect of the so called "digital divide") not because of the lack of technology but to its excessive complexity [1]. For example, in many cases, elderly people have difficulties in interacting with their own home appliances (including mobile and e-health devices [2][3]) and they prefer to ask for help to more skilled relatives (or not to use these devices at all). While this situation is annoying for most 
users, it could even represent a barrier for people with impairments; in fact, often it is the surrounding ICT environment - rather than the nature of their own impairments that tends to disable them, reducing their possibilities to access information and services.

In this paper we illustrate a novel technology called Mobile Electronic Memo (MEM) whose purpose is to overcome the limitations imposed by the current handling of electronic information. A MEM attempts to achieve this goal by decoupling the information it carries from its physical and practical limitations. We explain this underlying principle and the relevant benefits it might bring in the next section. In section 3 we take a closer look at the design of MEMs, providing a description of a prototype implementation that has been realized as a proof of concept. Moreover, we hint at security and trust aspects, illustrating one key feature that has been implemented and experimented in our prototype: signature of a MEM using an Universal Integrated Circuit Card (UICC). We conclude this paper with a discussion of related works and technologies, explaining why, in our opinion, some of these technologies could be good candidates to implement MEMs as an industry-wide standard.

\section{Benefits of a Pure Information Centric Model}

Web pages, business cards, short messages and GPS Point of Interests: all of them are examples of electronic information. But why should they be primarily thought in terms of readable text instead of Braille dots or spoken words?

In its Italian essay [4], Riva describes media under three main aspects: the physical aspect, the pragmatic aspect and the symbolic aspect. The physical aspect refers to the natural properties of the media (hardware, interfaces) and it is strongly related to the pragmatic aspect, which denotes the people's interaction modalities with the media. The symbolic aspect finally defines the meaning received by the people, i.e. the semantics. In traditional media there are tight constraints among these three aspects and this represents one reason that explains why we are used to thinking at a web page or a business card as something readable, rather than e.g. tactile.

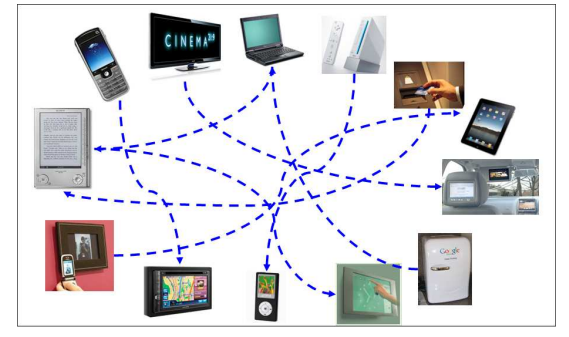

$A$

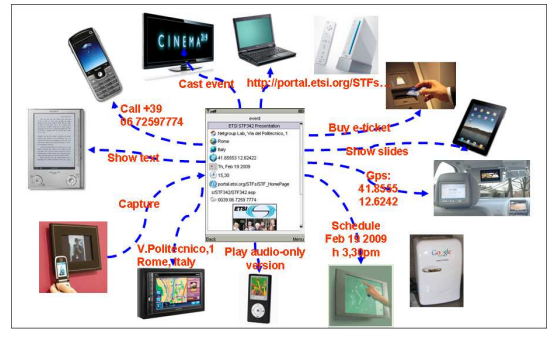

$B$

Fig. 1 From "spaghetti" information exchanges (a) to an information centric model (b).

On the contrary, making looser these constraints would bring two classes of benefits: firstly, development of multimodal and "assistive" technologies would be 
greatly simplified, being the same electronic information decoupled from specific devices and technologies; in the spirit of the "design for all" principles [5], even the word "assistive" could be no longer needed, because the information would be solely thought in terms of its semantics instead of its rendering techniques. Secondly the "spaghetti" information exchanges model depicted in (Fig. 1a) would disappear in favor of an electronic information centric model (Fig. 1b), where standardized information, expressed as MEMs, could be automatically exchangeable and interoperable across many different devices and appliances, through a number of different communication technologies.

\section{Mobile Electronic Memos}

A Mobile Electronic Memo (MEM) does not assume a specific physical support and interaction mechanism, but represents the semantics of the information it carries in a neutral way, using a machine understandable representation (i.e. an ontology). In particular, it supports the following features:

1. it is a versatile container of information;

2. it is machine understandable - therefore independent from specific devices, user interfaces and languages;

3. it can be transported across different kinds of bearers and networks;

4. it supports different user interaction paradigms, depending on the device or appliance it is executed on.

From an end user's point of view, MEMs may be created or captured from several sources; examples include, but not limit to: capture from the environment [6] via Near Field Communication (NFC), RFID, Bluetooth; optical capture through a QR code, location-based capture with GPS coordinates used to "lookup nearby MEMs", retrieval from the Web or from a mass storage memory, etc.

Once created or captured, depending on their contents, MEMs may be annotated, personalized, shared with other users or transferred to nearby devices. Users can browse created, received or captured MEMs using a variety of modalities.

MEM-aware devices and applications may provide several useful services based on the information MEMs contain: for example, a MEM aware navigator might drive the user to a location encoded in a captured MEM; a MEM aware smartphone might start a phone call or schedule an event in the user's agenda, using the information (telephone numbers, meeting details) contained in a received MEM; a MEM aware TV handset might auto-configure its settings taking their values from the information contained in a MEM, etc.

\subsection{MOVE}

Despite the current lack of standard technologies able to fully support the concept of MEM, many of the aforementioned features have been experimented in a prototype system including MEM-aware software and devices. The prototype system, named Mobile Open and Very Easy (MOVE), has been developed in the context of the EU co-funded research project Simple Mobile Services (IST 2006 034620). 
MOVE is based on a modular architecture. The base modules, implemented in Java Microedition (Java ME), are the Core, the Middleware, the GUI and the Outdoor \& Indoor Navigator modules. In particular, the Middleware module consists of an abstract API that hides the underlying transport mechanisms, enabling devices running MOVE to pass messages using either the HTTP protocol or the SIP protocol.

The GUI module is based on a porting of Thinlet, a very efficient toolkit which uses a XML-based model for the GUI elements. MOVE extends the original porting in order to provide an easier object oriented toolkit for GUI programming, fitting the different features and interaction modalities of a variety of devices. The Outdoor \& Indoor Navigator module provides maps and navigation, and a collection of functionalities to search a place, to search a route, to get GPS (or approximate) positions. Many of its functionalities are provided by the open source Open Street Map platform - which however offers a quality of the service not geographically uniform: coverage is better in large cities, and variable - depending upon the specific country - in small cities, villages and the countryside.

In order to port MOVE over different mobile platforms, including Symbian, Android, BlackBerry, and Windows Mobile, MOVE uses its own building tool that integrates the open source J2ME Polish Janus toolset. Third party programmers can develop additional modules for MOVE either using Java ME or platform specific languages. Modules developed using platform specific languages are wrapped into Java ME classes in order to provide unified interfaces to the rest of the framework. The communication with a platform specific API is usually realized using local IP sockets.

The full list of features offered by MOVE together with the list of supported platforms is provided in [7].

\subsection{A Proof of Concept}

In the following we illustrate part of the Simple Mobile Services project trial which consisted in using MEMs and the MOVE framework to announce a real event to different groups of people in the campus of the University of Rome Tor Vergata. This trial was intended to demonstrate how interoperability among several different technologies could be easily achieved by using MEMs; accessibility and multimodality were not particularly emphasized, but, as it has been observed, the highly degree of interoperability shown during this trial could be a key feature to foster the take-up of accessible and multimodal products and services based on MEMs.

In February 2009, two ETSI experts presented their standardization activity on "Personalization and User Profile Management" to researchers, post-docs and PhDstudents at the University of Rome Tor Vergata. This presentation was also advertised by distributing MEMs to invited participants. Some participants received the invitation through an email message containing an hyperlink to the MEM. Others received the MEM in their smart phones (which were running MOVE) through an instant messaging application. The organizers also exposed a physical poster which contained details about the presentations and a $\mathrm{QR}$ code. Capturing the $\mathrm{QR}$ code through the phone camera triggered the advertising MEM to be downloaded directly 
into the phone's memory. Some students were invited to diffuse the invitation to friends by transferring the MEM between their phones using Bluetooth, NFC or memory cards.

Mobile phones and laptops stored the received MEM as a file in their local filesystems. The file was displayed on their screens as a traditional hypertext containing information about the presentation. However other kinds of media enrichments (e.g. audio clips) could have been included in the MEM, making it suitable for running on other devices (e.g. portable audio/video players, gaming devices, etc.)

The advertising MEM included a phone number to dial in order to obtain further information, the date, time and duration of the presentation and the GPS coordinates of the location where the presentation was going to take place. By clicking on the displayed phone number, phones were able to initiate a call toward that number. Opening the MEM in a (MEM-aware) calendar application triggered the information contained in the MEM to be converted into an iCal event suitable to be automatically scheduled into the users' agenda. Finally, using Bluetooth the MEM could be transferred to a navigator appliance ${ }^{1}$. The navigator responded to this action setting the received MEM as a new Point of Interest (PoI) assumed to represent the next planned destination.

\subsection{Trust in Mobile Electronic Memos}

Given the very wide scope they cover and the possible presence of sensitive information inside MEMs, security and trust are one of the primary concerns that have been considered in designing MEMs. As an example, in order to prevent users from spam and phishing, MEMs or part thereof may be encrypted and digitally signed by the author (or the sender), allowing the recipient to verify their trustworthiness. In addition, one could think at this digital signature as a tool to implement nonrepudiation capability, thus making MEMs suitable for proof of purchases and subscriptions.

In order to fit these goals, not only service providers but also end users should be able to sign and encrypt their own MEMs; one way to implement this requirement could be through UICC functionalities [8]. Real UICCs featured with Javacard applets were used in the IST-SMS trial to implement the scenario depicted in Fig. 2, where two users exchange a trusted MEM. User A is asked to sign the MEM she is sending because a policy defined by user B does not allow the acceptance of untrusted MEMs. User A enters her PIN code in order to access the key-ring contained in her phone's UICC. Then, her device signs the MEM. Finally, the MEM is sent to user B which can browse its trusted content.

Obviously some transactions could employ lighter weight security mechanisms, in order to avoid the overhead due to the handing of certificates and keys (for example, a digest may be sufficient to prove the integrity of the information when the MEM is sent via a slow, but trustworthy channel).

\footnotetext{
${ }^{1}$ The navigator was emulated using a smart phone equipped with a navigation software.
} 
Since the present paper is mainly intended to provide an overview of the concept of MEM, we omit to describe other technical options; however, the reader could find lots of interesting technical details in a public available technical report about MEMs and their prototype implementation [9].
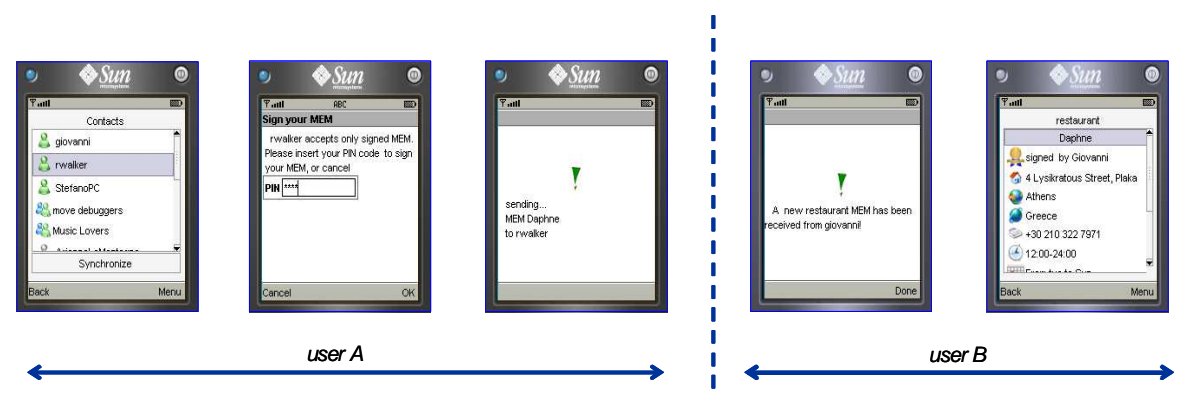

Fig. 2 Securing a MEM using UICC signature.

\section{Related Works}

There exist several standard formats designed for information exchanges and supported across different kinds of devices (mainly computers and mobile phones); Few examples include:

1. vCard [10], the consolidated standard electronic format for business card,

2. iCal [11], a widely supported file format allowing users to send meeting requests and tasks to other users,

3. XAL [12] and PIDF [13], respectively OASIS and IETF standards for codifying addresses and geographic locations...

However, these formats are typically limited to bits of information intended for specific purposes, e.g., exchanging information about a specific place or an event. Nevertheless, single versatile containers for various types of information are under investigation in many standardization fora. For example, both the Microformats Forum [14] and the MPEG-21 Group, pursuing different goals, are standardizing flexible formats to support the structured aggregation of different kinds of information resources. MPEG-21 explicitly provides support for alternative modes of presentation of digital resources (images, text, audio, etc.). It also specifies tools to support accessibility of digital items to various users, including users with audio-visual impairments [15].

\subsection{Semantic Web Technologies}

As the main finding behind the idea of MEM is to express information in a machine readable format, we now provide a brief review of related works based on semantic technologies. Ontologies are not new in computer science, however they 
have been traditionally limited to specific environments (reasoning and logics) or applications (the Semantic Web and Linked Data).

Their usage as a "neutral" vehicle to convey electronic information among heterogeneous devices is a relatively new topic of research. Actually this topic has been recently investigated in the context of the ARTEMIS-JU SOFIA (Smart Objects For Intelligent Applications) research project and in the Finnish national research projects DIEM (Device Interoperability EcosysteM) [16][17]. The two projects have produced a platform called SMART-M3 which combines distributed networked systems with the Semantic Web, enabling devices and software entities to exchange information conforming to common ontology models.

A second promising direction of research is semantic annotation of already existing formats. RDF-a [18] is a recent proposal to augment Web pages with RDF statements, turning them into richer container of semantic information which can then be understood and automatically processed by software. RDF-a is currently used with Web pages and within Web browsers. Its combination with other file formats spanning across different devices and appliances (e.g. MPEG-21) is unexplored and, we suggest, could represent a good candidate for the implementation of the concept of MEM.

\subsection{Machine-to-Machine Interactions}

Fitting the goal of a common file format and of consolidated semantics might be not enough, as the interoperability outside the boundary of the Web could be harder to achieve; this is mainly because of missing common information presentation facilities and the lack of standards for the transport of the information. Then, even if one could be tempted to rely always on Web-based solutions, there are many practical cases in which these solutions are not viable. This occurs, for example, when:

1. The information is better transmitted - over a relatively long range - in a peer-topeer fashion, using e.g. a short message or a MMS;

2. Many bearers are available and it is preferable to use a minimum range connection technique (e.g. PAN, or even transfer by USB sticks and memory cards), as this might have a major impact on performances and energy efficiency;

3. Short range connections are the only transmission mechanism (e.g., when communicating with devices without Internet connection, such as navigators, home or car appliances, portable media players, etc.);

4. Transfer over memory cards is the only transmission mechanism (e.g., when in "flight mode")

5. Users might be reluctant to post privacy sensitive information on the Web, as at today there is no effective solution to the "digital forgetting" problem (sensitive or inappropriate information posted on the Internet could hunt one forever).

The ongoing standardization work at ETSI Machine-to-Machine Technical Committee (M2M TC) is trying to address these situations by specifying a framework supporting transmission of information on a variety of devices and protocols. In Table 1 we list several requirements collected for a MEM aware system, together with corresponding capabilities of a M2M system [19] matching these requirements. 
Table 1. MEM requirements and M2M capabilities.

\begin{tabular}{l}
\hline Requirement \\
\hline Several different devices (smartphones, \\
navigators, media players, personal \\
organizers, home/office appliances, incar \\
devices), should be supported. \\
An uniform identification mechanism for \\
end users, devices and groups is needed. \\
Various forms of asynchronous and \\
unsolicited communications should be \\
supported; "Autoplay" should be allowed. \\
Various forms of delivery (anycast, \\
unicast, multicast, broadcast) should be \\
supported. \\
It should be possible to transmit MEMs \\
over a large set of bearers (including short \\
range connectivity and "delay tolerant \\
links", e.g. memory cards).
\end{tabular}

Multimedia should be supported.

Outdoor localization should be supported.

Indoor localization should be supported.

It should be possible to "capture" a MEM from the surrounding environment, for example using NFC, RF-ID, QR codes or Bluetooth.

MEMs may implement spam/phishing prevention, non-repudiation capability, confidentiality, proof of purchases and subscriptions.

Some use cases for MEMs may involve charging (e-Tickets, pass tokens, etc.)
M2M corresponding capability

A M2M system natively supports a wide range of different devices.

The M2M system allows flexible addressing schemas.

The M2M system provides a wide range of message delivery modes. It even provides delivery towards sleeping devices.

The M2M system provides message exchanges across multiple communication paths, based on policy rules and taking into account charging, privacy, energy saving, performances.

The M2M system provides telecom capabilities exposure (localization, subscription configuration, authentication, reachability information, messaging and IMS access).

Sensors connected to the M2M system could provide information to be included into a MEM (position, temperature, speed, light intensity, pressure,...) or they could provide a way to retrieve a whole MEM (e.g. reading a visual code, detecting an RF-ID,...).

The M2M system shall provide a secure and trusted environment (based on, e.g., the UICC) for security critical operations.

The M2M system shall support micropayments. 


\section{Conclusions}

In this paper we presented the concept of Mobile Eelectronic Memo (MEM), a versatile, machine understandable "aggregator" of information. Unlike other media, a MEM does not impose any particular physical or pragmatic constraint and focus only on the symbolic aspect of the communication. Technically, this is made possible by replacing human readable contents with a semantic description of the information. Major benefits brought by this approach include interoperability and accessibility.

Despite a prototype system (including some basic security features) has been implemented, at the time of writing there is no standard framework that could fully support MEMs; nevertheless we believe that a combination of existing XML/RDF based languages and technologies under development for machine-to-machine systems could provide a valuable opportunity for allowing MEMs to evolve into an industry-wide standard.

Acknowledgments. We would like to thank all people that contributed to the development of MEMs in the context of the EU co-funded research project Simple Mobile Services. In particular we thank N. Blefari Melazzi, project coordinator, R. Glaschick (Siemens C-Lab) for his key contributions on security, privacy and trust aspects and C. Rust (Sagem Orga) and A. Rabbini (Telecom Italia) for having provided specially designed UICCs supporting MEM encryption and signature. Last but not least we thank ETSI members for the valuable feedback received during the presentation of our work to the ETSI Human Factors Technical Committee. Current activities on MEMs at University of Rome Tor Vergata are supported by CREALAB and ErgonixART in the context of the POR FESR project "wetourist" funded by FILAS/Regione Lazio. 


\section{References}

1. Klironomos, I., Antona, M., Basdekis, I., Stephanidis I.: Promoting Design for All and eAccessibility in Europe. Universal Access in the Information Society. Volume 5, Number 1, 105-119, DOI: 10.1007/s10209-006-0021-4

2. Istepanian, R.S.H., Lacal, J.C.: Emerging mobile communication technologies for health: some imperative notes on m-health. Engineering in Medicine and Biology Society. Proceedings of the 25th Annual International Conference of the IEEE, September 17-21, 2003, DOI: 10.1109/IEMBS.2003.1279581

3. Hubert, R.: Accessibility and usability guidelines for mobile devices in home health monitoring. ACM SIGACCESS Accessibility and Computing (2006)

4. Riva, G.: Psicologia dei nuovi Media. Il Mulino, Bologna (2004)

5. The Center for Universal Design: The Principles of Universal Design, Version 2.0. North Carolina State University, Raleigh, NC (1997)

6. Herting, T., Broll, G.: Acceptance and Usability of Physical Mobile Applications. Mobile Interaction with the Real World (MIRW 2008), Workshop at the 10th International Conference on Human-Computer Interaction with Mobile Devices and Services (MobileHCI 2008), Amsterdam, the Netherlands, September 2, 2008, ISBN 978-3-8142-2134-2

7. Salsano, S., Bartolomeo, G.: MOVE, Thinlet and HECL, Technical Report, available at http://netgroup.uniroma2.it/SMS/TechnicalReports

8. Rust, C., Salsano, S., Schnake, L.: The SIM card as an Enabler for Security, Privacy, and Trust in Mobile Services, ICT-MobileSummit 2008, Stockholm, Sweden, June 10-12, 2008, ISBN: 978-1-905824-08-3

9. Salsano, S., Bartolomeo, G., Glaschick, R., Walker, R., Blefari-Melazzi, N.: Mobile Electronic Memos for Simple Mobile Services. Technical report, IST-SMS project (2008)

10.Dawson, F., Howes, T.: RFC 2426 - vCard MIME Directory Profile, IETF (1998)

11.Dawson, F., Stenerson, D.: RFC 2445 - Internet Calendaring and Scheduling Core Object Specification (iCalendar), IETF (1998)

12.Customer Information Quality Technical Committee: extensible Address Language (xAL) v2.0. Technical specifications, OASIS (2004).

13.Sugano, S., Fujimoto, G., Klyne, G., Bateman, A., Carr, W., Peterson J.: RFC 3863 Presence Information Data Format (PIDF), IETF (2004)

14.The Microformats community, http://microformats.org/

15.Vetro, A.: MPEG-21 Digital Item Adaptation: Enabling Universal Multimedia Access. IEEE Multimedia, vol. 11, no. 1, pp. 84-87, Jan. 2004, doi:10.1109/MMUL.2004.1261111

16.Liuha, P., Lappeteläinen, A., and Soininen, J-P.: Smart Objects for Intelligent Applications first results made open, ARTEMIS Magazine, October 2009, No. 5, pages 27-29.

17.Koljonen, T.: ARTEMIS and the rest of the world, ARTEMIS Magazine, October 2009, No. 5, pages 30-31.

18.Birbeck, M., Pemberton, S., Adida, B.: RDFa Syntax: A collection of attributes for layering RDF on XML languages, W3C (2008)

19.Machine to Machine Technical Committee: TS 102689 v1.1.1 Machine to Machine communications (M2M); M2M service requirements. Technical specifications, ETSI (2010) 